\title{
Originalartikel
}

\section{Zur Wirkungsweise von Ameisensäure bei Varroa jacobsoni Oud und der Honigbiene (Apis mellifera L)}

\author{
HK Bolli 1, S Bogdanov 1, A Imdorf 1, P Fluri 2* \\ ${ }^{1} \mathrm{CH}-5035$ Unterentfelden, Flurweg 6; \\ ${ }^{2}$ Eidgenössische Forschungsanstalt für Milchwirtschaft, Sektion Bienen, \\ CH-3097 Liebefeld-Bern, Schweiz
}

(Eingegangen 14 September 1992, angenommen 22 Oktober 1992)

\begin{abstract}
Zusammenfassung - Varroa-Milben, Bienenlarven und frisch geschlüpfte Bienen wurden in vitro und unter Praxisbedingungen im Bienenvolk mit dem natürlichen Akarizid Ameisensäure bedampft und anschliessend die Atmung manometrisch anhand des Sauerstoffverbrauchs gemessen. Ameisensäure (ca 2500 ppm in Luft) hemmte in vitro die Atmung der Milben und auch der jüngerer 2tägigen und älteren 4-tägigen Bienenbrut unterschiedlich schnell bis zur Vollständigkeit, die der jungen Bienen nur teilweise: Die Milben mit ihrer geringsten Körpermasse und dabei relativ grössten Körperoberfläche, verbunden mit hoher Atmungsintensität, reagierten am empfindlichsten, dann folgten die 2-tägigen, dann die 4-tägigen Arbeiterinnenlarven. Mit der Atmungshemmung einher ging eine Uebersäuerung des Körpers. Verätzungen oder Nekrosen konnten jedoch nicht festgestellt werden. Unter Praxisbedingungen im Bienenvolk ( $\leq 500 \mathrm{ppm}$ Ameisensäure in Luft) konnte nur noch die Atmung der Milben gehemmt werden. Die Ergebnisse werden diskutiert.
\end{abstract}

Varroa jacobsoni / Apis mellifera / Ameisensäure / Atmung / in vitro Untersuchung / Akarizid

\section{EINLEITUNG}

Seit den ersten Berichten über einen möglichen Einsatz von Ameisensäure (= AS) zur Bekämpfung der Varroatose (Künzler et al, 1979; Ritter and Ruttner, 1980) erlangte dieses natürliche Akarizid vor allem wegen seiner sehr geringen Resistenzproblematik zunehmend an Bedeutung über den Wirkungsmechanismus und die
Gründe für die Selektivität im Kontakt mit Milben und Bienen ist jedoch wenig bekannt. Von akuten Intoxikationen beim Menschen wissen wir, dass es nach Über lastung der Eliminations- und Metabolisierungskapazität zu einer Akkumulation von AS in den Zellen kommt. Die dabei eintretende Azidose führt $\mathrm{zu}$ pathologischen Veränderungen im Metabolismus und hemmt auch, sehr wahrscheinlich über

\footnotetext{
* Correspondenz und Sonderdrucke
} 
Cytochrom c, die Zellatmung (Nicholls, 1975; Petersen, 1977; Liesivuori und Savolainen, 1991). Auch die toxischen Effekte von AS auf das Zentralnervensystem von Ratten sollen letzlich auf der gedrosselten oxydativen Energieproduktion beruhen (Savolainen und Zitting, 1980; Sommer, 1988).

In der vorliegenden Arbeit wurde daher der Einfluss von AS auf die Atmung von Varroa-Milben, Bienenlarven und frisch geschlüpften Bienen untersucht. Parallel dazu wurden die Körpersäfte der mit AS bedampften Tiere einem $\mathrm{pH}$-Indikatortest unterzogen und ihre Gewebe, speziell die Atmungsorgane, auf das Vorliegen von Verätzungen und Nekrosen untersucht.

\section{MATERIAL UND METHODE}

\section{Bedampfung der Versuchstiere mit Ameisensäure (= AS) in vitro}

In ein Glasgefäss von 4,8 I Inhalt wurden $30 \mathrm{ml}$ $60 \%$ ige AS auf eine saugfähige Bodenplatte ausgebracht und in $3 \mathrm{~cm}$ Abstand darüber ein Gitter gelegt. Die aus den Zellen entnommenen Bienenlarven (Arbeiterinnenbrut) kamen in Schälchen, frisch geschlüpfte Arbeiterinnen und die aus Arbeiterinnenbrut gewonnenen VarroaWeibchen in luftdurchlässigen Käfigen auf das Gitter zu liegen. Das Glasgefäss wurde während der Expositionszeiten von 13 bis $360 \mathrm{Min}$ zugedeckt gehalten und bei Zimmertemperatur aufgestellt. Unter diesen Bedingungen herrschte im Gefäss eine AS-Konzentration (gaschromatographisch bestimmt) von ca $2500 \mathrm{ppm}$, die Luftsättigung von ca 30000 ppm wurde also nicht erreicht.

\section{Bedampfung der Versuchstiere mit Ameisensäure im Bienenvolk}

Bei Bienenvölkern mit einer Brutzarge wurde eine mit $20 \mathrm{ml}, 30 \mathrm{ml}$ oder $40 \mathrm{ml} 60 \%$ ige AS getränkte Platte über die Rahmen gelegt. Die Kon- zentration in der Mitte des Brutnestes stieg bei dieser Applikationsweise kaum über 500 ppm (Adelt und Kimmich, 1986; Charriere et al, 1992).

Untersucht wurde die im Volk vorhandene Arbeiterinnenbrut, ferner gekäfigte Milben, die aus der Brut anderer Völker gesammelt und zwischen die Waben gehängt wurden, sowie die freien Milben des Versuchsvolkes, die anlässlich der Bedampfung von den Bienen abfielen. Bei Langzeitversuchen mit Larven musste korrekterweise die mögliche Auswirkung einer mangelhaften Brutpflege durch die mitbedampften Pflegebienen bedacht werden. Bei einem 12stündigen Bedampfungsversuch im Volk wurden daher Larven auf einem Wabenteil sowohl im Versuchs- als auch im Kontrollmagazin mit Hilfe eines Gitters für die Pflegebienen unzugänglich gemacht und mit normal gepflegten Kontrollarven verglichen.

\section{Messung der Atmung}

Petrova et al (1982) untersuchten die Atmung von Varroa-Milben mit manometrischen Methoden erfolgreich mit einem speziell kleinen Warburg-Gefäss von $2 \mathrm{ml}$ Inhalt. Für unsere Messungen wurden normale Warburg-Gefässe von ca $12 \mathrm{ml}$ Inhalt mit entsprechend grösserem Milbenbesatz verwendet.

Der Sauerstoffkonsum der Versuchstiere wurde anschliessend an die Bedampfung mit AS bei konstantem Volumen nach Warburg bei $34{ }^{\circ} \mathrm{C}$ gemessen und mit demjenigen von unbedampften Tieren (Kontrollen) verglichen.

Pro Gefäss wurden entweder 4 viertägige oder 10 zweitägige Bienenlarven, 3 frisch geschlüpfte Arbeiterinnen oder 50 Milben in den Hauptraum plaziert. Zur Elimination des Kohlendioxids aus dem Gasraum wurde Kaliumhydroxid $(0,2 \mathrm{ml} 20 \%)$ entweder in den zentralen Einsatz oder in die für die Tiere unzugänglich gemachte Birne (bei Milben und adulten Bienen) zugefügt. Aus messtechnischen Gründen wurde bei den Milben noch ein mit $0,2 \mathrm{ml}$ Wasser getränktes Filterpapierchen in den Einsatz gesteckt. Es zeigte sich, dass die geringe Körpermasse der Milben allein nicht ausreicht, um durch inre Wasserabgabe den Wasserdampfdruck in den Gefässen stabil zu halten. Die Versuche wurden zwei- bis dreimal durchgeführt. In den Abbildungen stellt somit jeder Punkt in einer 
Kurve ein arithmetisches Mittel dar, gewonnen aus 2 bis 3 Messungen. Um einen Anhaltspunkt über die Streuung $\mathrm{zu}$ vermitteln, sind in $\mathrm{Abb} 1$ auch noch die zugehörigen Messwerte eingezeichnet. Ein Vergleich der vorliegenden an unbehandelten Tieren gemessenen Atmungsraten mit solchen, die von anderen Autoren ermittelt wurden, so an Varroa-Milben bei Petrova et al (1982), an Bienenlarven verschiedenen Alters bei Melampy und Willis (1939), an ruhenden Arbeiterinnen bei Blanke und Lensing (1989), letztere mit einem IR-Analysator arbeitend, lassen ähnliche Grössenordnungen erkennen.

\section{pH-Indikatortest und Untersuchung auf Gewebeschäden}

Nach den Atmungsmessungen wurden die Tiere im Mörser zerrieben und ihr Körpersaft mit einigen Tropfen Wasser und dem Indikator Bromkresolpurpur (Umschlagsbereich $\mathrm{pH} \mathrm{6,8-4,8)} \mathrm{vesetzt.}$ Durch den Farbumschlag von purpur zu gelb wurde auf das Vorliegen einer allgemeinen Übersäuerung geschlossen. Weitere Tiere wurden speziell im Bereich der Stigmen und Tracheen nach Verätzungen oder nekrotischen Veränderungen untersucht. Dies geschah mit Hilfe von Frischpräparaten unter dem Lichtmikroskop oder die Tiere wurden über eine Kritisch-PunktTrocknung oder eine Vakuum-Gefriertrocknung und anschliessende Beschichtung im Sputtergerät für das Rasterelektronenmikroskop vorbereitet.

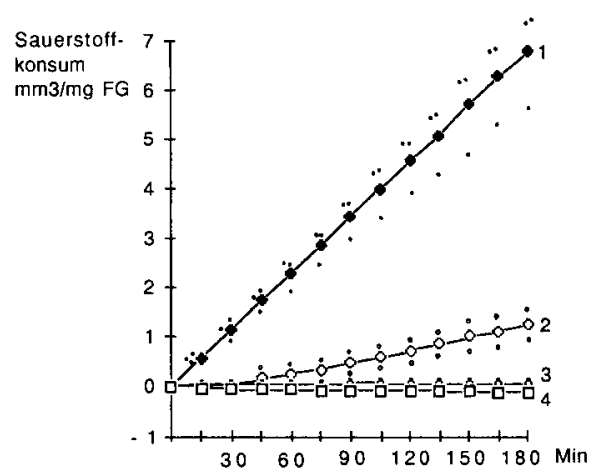

Abb 1. Sauerstoffkonsum von 2 Tage alten Bienenlarven nach Bedampfung mit AS in vitro (mittleres Gewicht/Larve $=18,5 \mathrm{mg}$ ). 1: unbedampft (Kontrolle). $n=3$. 2: $40 \mathrm{Min}, 3: 120 \mathrm{Min}$, $4: 360$ Min bedampft. $n=2$.

\section{ERGEBNISSE}

\section{Bedampfung der Versuchstiere mit Ameisensäure in vitro}

In den Abbildungen 1 und 2 ist der Sauerstoffkonsum nach verschieden langen $\mathrm{Be}$ dampfungen bei 2 resp 4 Tage alten Arbeiterinnenlarven dargestellt. Der auf das gleiche Frischgewicht (=FG) bezogene Sauerstoffkonsum liegt bei den unbehandelten 2 Tage alten Larven mit $2,25 \mathrm{~mm}^{3} / \mathrm{mg} \mathrm{FG} /$ Std fast doppelt so hoch wie bei den 4 Tage alten Larven. Die jungen Larven reagierten gegenüber AS wesentlich empfindlicher als die älteren. Bereits nach $120 \mathrm{Min}$ war die Atmungshemmung total. In Abb 3 ist ein entsprechender Versuch mit frisch geschlüpfen Arbeiterinnen dargestellt. Diese Bienen verloren ihre normale Lokomotorik während der Bedampfung bereits nach etwa 25 Min, nach 40 Min bewegten sie sich nur noch schwach. Trotzdem war ihr Sauerstoffkonsum noch unvermindert, erst ab 120 Min Bedampfung begann sich eine Hemmung abzuzeichnen. Nach 24 Stunden Erholungszeit bewegten sich nur noch die unbedampften Kontrollbienen.

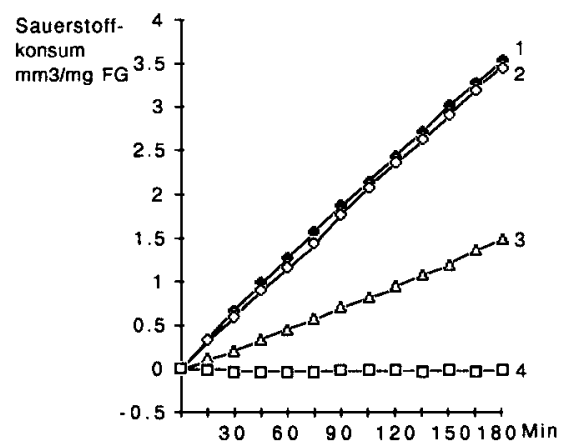

Abb 2. Sauerstoffkonsum von 4 Tage alten Bienenlarven nach Bedampfung mit $A S$ in vitro (mittleres Gewicht/Larve $=63 \mathrm{mg}$ ). 1: unbedampft (Kontrolle). $n=3.2: 40 \mathrm{Min}, 3: 120 \mathrm{Min}$, $4: 360$ Min bedampft. $n=2$. 
Noch empfindlicher als die zweitägigen Bienenlarven reagierten die Varroa-Milben (Abb 4). Nach 13 Min Bedampfungsdauer bewegten sich die Milben zwar noch spontan, ihr Sauerstoffkonsum war jedoch auf etwa ein Viertel gesunken, nach $40 \mathrm{Min}$ Bedampfungsdauer war die Atmungshemmung bereits vollständig.

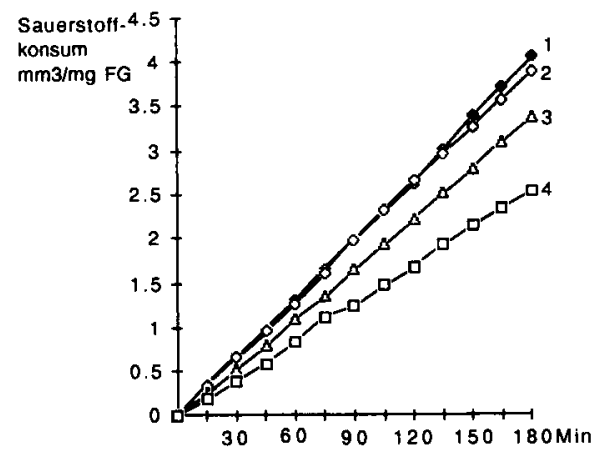

Abb 3. Sauerstoffkonsum von frisch geschlüpften Arbeiterinnen nach Bedampfung mit $A S$ in vitro. $n=3$. 1: unbedampft (Kontrolle). 2: 40 Min, $3: 120 \mathrm{Min}, 4: 360 \mathrm{Min}$ bedampft.

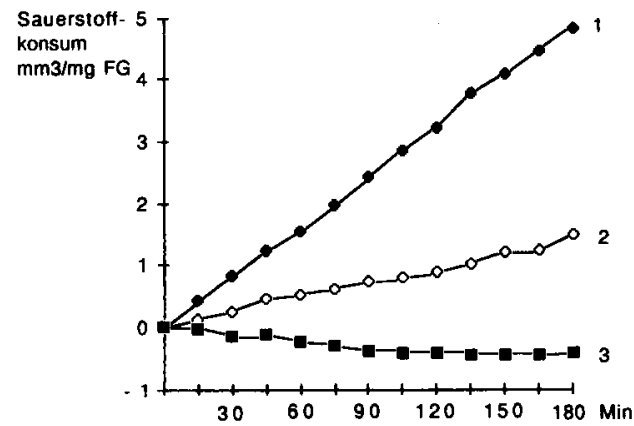

Abb 4. Sauerstoffkonsum von Varroa-Milben nach Bedampfung mit $A S$ in vitro. $n=2$. 1 : unbedampft (Kontrolle). 2: 13 Min, $3: 40$ Min bedampft.

\section{Bedampfung der Versuchstiere mit Ameisensäure im Bienenvolk}

In Abb 5 ist der Einfluss von AS auf die Atmung der Bienenbrut nach dreistündiger Bedampfung eines Bienenvolkes dargestellt.

Ein deutlicher Hemmeffekt war bei diesen geringeren AS-Konzentrationen nicht mehr erkennbar. Das Ergebnis eines Langzeitversuches mit 2-tägigen Bienenlarven ist in Abb 6 dargestellt. Die während 12 Stunden nicht mehr gepflegten, weil abgesperten, Kontrollarven wiesen im Vergleich zu den gepflegten eine deutlich geringere Atmungsrate auf, während zwischen den ungepflegten Kontrollarven und den mit AS bedampften Larven kaum Unterschiede auftraten. Somit ist auch hier kein Hemmeffekt durch die AS erzielt worden.

Anders fielen die Ergebnisse der Milbenversuche aus. Nach dreistündiger Bedampfung wurde die Atmung bei den im Brutnest gekäfigten Milben grossenteils, bei den von den Bienen abgefallenen Milben schwächer gehemmt (Abb 7). Die

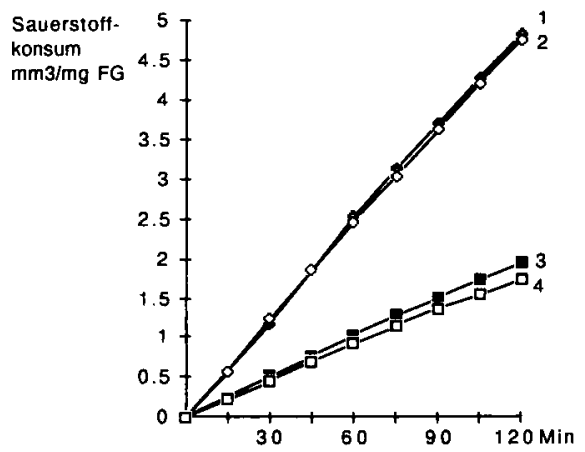

Abb 5. Sauerstoffkonsum von Bienenlarven nach 3-stündiger Bedampfung mit $40 \mathrm{ml} \mathrm{AS}$ im Volk. A. Larvenalter 2 Tage (mittleres Gewicht/ Larve $=7.0 \mathrm{mg}$ ). $n=3.1$ : unbedampft (Kontrolle), 2: bedampft. B. Larvenalter 4-5 Tage (mittleres Gewicht/Larve $=109 \mathrm{mg}$ ). $n=2$. 3: unbedampft (Kontrolle). 4 : bedampft. 


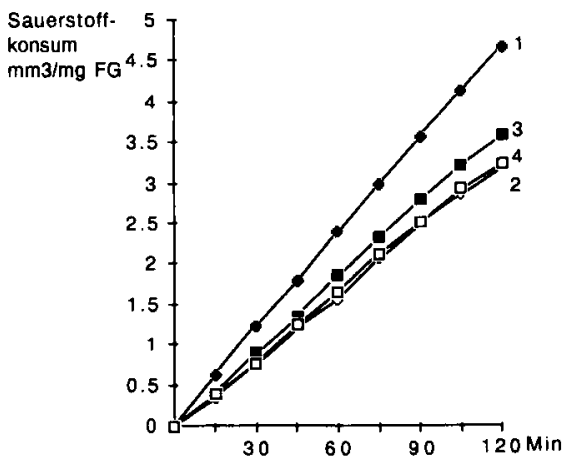

Abb 6. Sauerstoffkonsum von 2 Tage alten Bienenlarven (mittleres Gewicht/Larve $=6.1 \mathrm{mg}$ ) nach 12-stündiger Bedampfung mit $40 \mathrm{ml}$ AS im Volk. $n=2$. A. Larven für die Pflegebienen zugänglich. 1: unbedampft (Kontrolle), 2: bedampft. B. Larven für die Pflegebienen unzugänglich. 3: unbedampft (Kontrolle). 4: bedampft.

gefallenen Milben waren im Gegensatz zu den gekäfigten noch grösstenteils bewegungsfähig.

\section{pH-Indikatortest und Gewebeschäden}

Alle bedampften Tiere mit vollständiger Atmungshemmung, sowohl Bienenlarven wie Milben, die dem Indikatortest unterzogen wurden, waren übersäuert. Ältere Arbeiterinnen, die zum Vergleich mitgetestet wurden, waren rascher übersäuert als frisch geschlüpfte. Verätzungen oder $\mathrm{Ne}$ krosen liessen sich anhand der durchgeführten mikroskopischen Untersuchungen nicht feststellen.

\section{DISKUSSION}

Ameisensäuredämple können je nach Konzentration und Einwirkungsdauer die Atmung sowohl von adulten Bienen und ihrer Brut als auch von Varroa-Milben

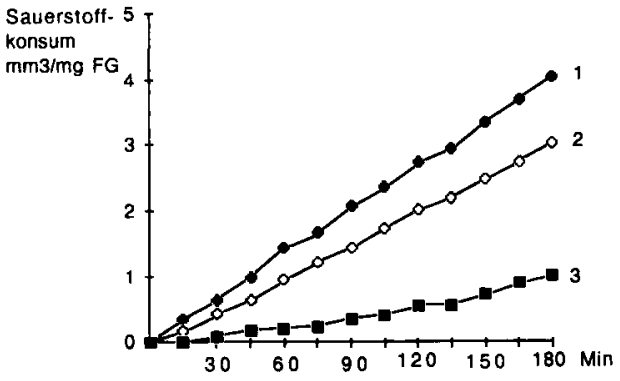

Abb 7. Sauerstoffkonsum von Varroa-Milben nach 3-stündiger Bedampfung mit $20 \mathrm{ml} \mathrm{AS}$ im Bienenvolk. $\mathbf{n}=2$. 1: unbedampft (Kontrolle). 2 : bedampft, Milben frei auf den Bienen. 3: bedampft, Milben gekäfigt.

hemmen. Es zeigt sich eine gewisse Korrellation zwischen der Körpergrösse respektive der Atmungsintensität und der Empfindlichkeit der untersuchten Tiere bezüglich Atmungshemmung. Die Milben mit der von den untersuchten Tiergruppen geringsten Körpermasse und gleichzeitig relativ grössten Körperoberfläche (die Cuticula dürfte für Dämpfe etwas durchlässig sein), verbunden mit hoher Atmungsintensität, reagieren am empfindlichsten und sind auch am schnellsten übersäuert, es folgt die jüngere, dann die ältere Bienenbrut und schliesslich die am wenigsten empfindlichen frisch geschlüpften Arbeiterinnen. Offenbar wird bei den Milben die Puffer- und die Metabolisierungskapazität für AS rascher als bei den Larven oder bei den jungen Arbeiterinnen überschritten und die sich einstellende Gewebeübersäuerung könnte die Hemmung der Atmung auslösen. Die selektive Wirkung der AS auf die Varroa-Milben ist damit zumindest teilweise erklärbar. Die Beobachtung, dass Arbeiterinnen nach 40 Min Bedampfung 
zwar noch keine Atmungshemmung, jedoch bereits Störungen ihrer Lokomotorik aufwiesen von denen sie sich nicht mehr erholten, lässt allerdings noch neurotoxische Effekte vermuten. Diese können hier offensichtlich nicht auf eine verminderte oxydative Energieproduktion zurückgeführt werden, wie dies Savolainen und Zitting (1980) bei Ratten annehmen.

\section{DANKSAGUNGEN}

Herrn Dr M Müller, ETH Zürich, danken wir für den grosszügigen Zugang zum Elektronenmikroskop-Labor, Herrn Dr E Wehrli für seine Hilfe bei der Präparation, Herrn Prof E Brändle, Universität Bern, für fachliche Anregungen und Herrn Prof P Diehl, Universität Neuenburg, für die kritische Durchsicht des Manuskripts. Der Kanton Aargau ermöglichte durch seine finanzielle Unterstützung diese Arbeit.

\section{Summary - Action of formic acid on Varroa jacobsoni Oud and the honeybee (Apis mellifera L). In one series of experi- ments (in vitro tests), Varroa mites, honey- bee larvae of different ages and newly emerged worker bees were treated with rel- atively high concentrations of formic acid $(\approx$ 2500 ppm in the air). In another series of experiments honeybee colonies in hives were treated with $20-40 \mathrm{ml} 60 \%$ formic acid under practical beekeeping conditions ( $\leq 500 \mathrm{ppm}$ in the air). After both types of formic acid treatment the respiration of the animals was measured by the Warburg manometric method.}

In the in vitro tests formic acid inhibited oxygen consumption of the bee brood (figs 1,2 ), of newly emerged worker bees (fig 3) and of Varroa mites (fig 4). This indicates that the mites, but also young larvae with their small body size and intensive respiration react more sensitively to formic acid than older larvae and young bees.
Under conditions of formic acid treatments in bee hives there was no inhibition of the respiration of young and old larvae (fig 5). However, after prolonged formic acid treatments of bee colonies, a negative effect on brood care was observed. The resulting decrease in larval feeding might be the cause of the decrease in bee larvae respiration (fig 6). In the Varroa mites in a cage in the bee colony, respiration after one formic acid treatment was nearly arrested whereas the mites which fell from the bees during treatment showed only partial respiration inhibition (fig 7).

The bodies of the animals with inhibited respiration were found to be strongly acidic as tested by $\mathrm{pH}$ indicator. However, no necrosis or corrosive effects on the in vitro treated animals could be detected after examination with light and electron microscopy. The faster respiration inhibition of the Varroa mites could thus be partly due to their lower buffering and metabolizing capacity. This at least explains the selective effects of formic acid on Varroa mites, however, the existence of neurotoxic effects on both bees and mites seems possible.

Varroa jacobsoni / Apis mellifera / respiration / formic acid / acaricide / in vitro study

Résumé - Mode d'action de l'acide formique chez Varroa jacobsoni et l'abeille Apis mellifera. Dans une première série d'expériences, des acariens Varroa jacobsoni, des larves d'abeilles d'âges variés et des ouvrières fraîchement écloses ont été exposés in vitro, pendant des temps plus ou moins longs, à des vapeurs d'acide formique (AF) en concentrations relativement élevées (2 500 ppm dans l'air). Dans une seconde série, des colonies d'abeilles ont été traitées avec $20-40 \mathrm{ml}$ d'AF à $60 \%$ dans les conditions de la pratique apicole ( $\leq 500 \mathrm{ppm}$ dans l'air). La consommation 
d'oxygène des animaux a été mesurée par la méthode manométrique de Warburg après traitement de quelques heures. L'AF inhibe in vitro la respiration du couvain d'abeilles (figs 1 et 2 ), des ouvrières fraîchement écloses (fig 3 ) et des varroas (fig 4). Ces derniers, mais aussi les jeunes larves d'abeilles, dont la taille est faible et l'intensité respiratoire élevée, réagissent à l'AF de façon beaucoup plus sensible que les vieilles larves ou les jeunes abeilles adultes.

Après traitement des colonies d'abeilles dans les conditions de la pratique, la respiration n'est inhibée ni chez les jeunes larves ni chez les plus âgées (fig 5). Dans les essais de longue durée, il faut prendre en compte une influence possible d'une insuffisance de soins au couvain par les nourrices également traitées. Le manque de nourriture qui en résulte réduit aussi la respiration des larves (fig 6). Chez les varroas mis en cage dans la colonie, la respiration est presque totalement inhibée après un traitement, alors qu'elle ne l'est que partiellement chez les varroas qui se sont détachés des abeilles pendant le traitement (fig 7).

On a pu mettre en évidence, à l'aide d'un indicateur de $\mathrm{pH}$, une forte acidification des corps de tous les animaux dont la respiration était totalement inhibée. Pourtant l'examen en microscopie optique et éiectronique à balayage n'a détecté aucune nécrose, ni aucun effet corrosif chez les animaux traités in vitro. L'inhibition plus rapide de la respiration chez les varroas pourrait être due en partie à leur plus faible action de tampon et à une métabolisation réduite. Cela explique, au moins en partie, l'action sélective de l'AF sur les varroas, mais des effets neurotoxiques semblent possible aussi bien chez l'abeille que chez le varroa.

Varroa jacobsoni / Apis mellifera / acide formique / étude in vitro / respiration

\section{LITERATUR}

Adelt B, Kimmich KH (1986) Die Wirkung der Ameisensäure in die verdeckelte Brut. Allg Dtsch Imkerztg 12, 382-385

Blanke M, Lensing W (1989) Measurement of metabolic activity of the honeybee by assessing respiration. J Apic Res 28 (3), 131135

Charriere JD, Imdorf A, Kilchenmann V (1992) Konzentrationen der Ameisensäure in der Stockluft von Bienenvölkern während der Anwendung gegen Varroa jacobsoni. Allg Dtsch Imkerztg 26 (9), 12-16

Künzler K, Mook H, Breslauer H (1979) Untersuchung über die Wirksamkeit der Ameisensäure bei der Bekämpfung der Bienenmilbe Varroa jacobsoni. Die Biene 9, 372-373

Liesivuori J, Savolainen $H(1991)$ Methanol and formic acid toxicity biochemical mechanisms. Pharmacol Toxicol 69 (3), 157-161

Melampy RM, Willis ER (1939) Respiratory metabolism during larval and pupal development of the female honeybee (Apis mellifica L). Physiol Zool 12 (3), 302-311

Nicholls P (1975) Formate as an inhibitor of cytochrome $c$ oxidase. Biochem Biophys Res Comm 67 (2), 610-616

Petersen LC (1977) The effect of inhibitors on the oxygen kinetics of cytochrome $c$ oxidase. Biochem Biophys Acta 460 (2), 299-307

Petrova AD, Byzova YB, Tatsii VM, Emel'yanova OY (1982) Metabolic expenditures of Varroa jacobsoni - an ectoparasite of the honeybee. Translated from Dokl Akad Nauk SSSR, 262 (2), 499-502

Ritter W, Ruttner F (1980) Neve Wege in der Behandlung der Varroatose - Ameisensäure. Allg Disch Imkerztg 14 (5), 151-159

Savolainen H, Zitting A (1980) Glial cell effects of subacute formic acid vapour exposure. Acta Pharmacol et Toxicol 47 (3), 239-240

Sommer D (1988) Akute Intoxikationen durch Ameisensäure. Dissertation Medizin Fakultät Universität $\mathrm{CH}-8000$ Zürich 